\title{
Relaxation of dark matter halos: how to match observational data?
}

\author{
Anton N. Baushev ${ }^{1,2}$ \\ 1 DESY Zeuthen Platanenallee 6, 15738 Zeuthen, Germany \\ e-mail: baushev@gmail.com \\ 2 Institut für Physik und Astronomie, Universität Potsdam, 14476 Potsdam-Golm, Germany
}

Received 23 September 2013 / Accepted 25 June 2014

\section{ABSTRACT}

\begin{abstract}
We show that moderate energy relaxation in the formation of dark matter halos invariably leads to profiles that match those observed in the central regions of galaxies. The density profile of the central region is universal and insensitive to either the seed perturbation shape or the details of the relaxation process. The profile has a central core; the multiplication of the central density by the core radius is almost independent of the halo mass, in accordance with observations. In the core area the density distribution behaves as an Einasto profile with low index $(n \sim 0.5)$; it has an extensive region with $\rho \propto r^{-2}$ at larger distances. This is exactly the shape that observations suggest for the central region of galaxies. On the other hand, this shape does not fit the galaxy cluster profiles. A possible explanation of this fact is that the relaxation is violent in the case of galaxy clusters; however, it is not violent enough when galaxies or smaller dark matter structures are considered. We discuss the reasons for this.
\end{abstract}

Key words. dark matter - Galaxy: structure - Galaxy: formation - astroparticle physics - methods: analytical

\section{Introduction}

Although the dark matter (DM) component contributes most to the galaxy mass containment, a generally accepted explanation for some observational properties of the galaxy dark matter halos has not been supplied yet. In particular, there is some disagreement regarding the density profile in the center of the halos. Earlier $N$-body simulation suggested very cuspy profiles with an infinite density in the center (see, e.g., Moore et al. 1999; Neto et al. 2007). For instance, the Navarro-Frenk-White profile (hereafter NFW) behaves as $\rho \propto r^{-1}$ in this area. Recent simulations (Stadel et al. 2009; Navarro et al. 2010) favor an Einasto profile with a finite central density, but the obtained Einasto index $n$ is so high (typically $n \sim 5-6$ ) that the profile is very steep in the center and may still be called cuspy. Although the simulations mainly model the largest structures of the Universe, such as galaxy clusters $\left(M \sim 10^{15} M_{\odot}\right)$, their results are expected to be valid for smaller objects as well. Moreover, simulations of separate dark matter halos $\left(M \sim 10^{12} M_{\odot}\right)$ have also be performed (see, for instance, the Via Lactea project Diemand et al. 2007).

However, the correct interpretation of the $N$-body data requires a reliable estimation of the simulation convergence. The idea of $N$-body simulations is to substitute real tiny dark matter particles by heavy test bodies. Since there are fewer bodies, the task becomes computable. We face a problem, however: the test bodies collide much more effectively than the original DM particles. The strong encounters with high momentum transfer lead to evident effects such as kicking of the test particles from the halo. They are avoided in simulations by softening of the Newtonian potential near the test bodies. However, the gravitational force is long-acting, and the influence of weak long-distant collisions dominates. In a nutshell, the gravitational potential of homogeneous dark matter is plain, while the potential of the test bodies has local potential wells near the bodies, despite of the softening. This produces unphysical soft scattering of the bodies on each other and so leads to a collisional relaxation. The process can be described by the Fokker-Planck approximation (Landau $\&$ Lifshitz 1980). The characteristic time of the collisional relaxation is (Binney \& Tremaine 2008, Eq. (1.32)) $\tau_{r}=\frac{N(r)}{8 \ln \Lambda} \cdot \frac{r}{v}$, where $v$ is the characteristic particle speed at radius $r, N(r)$ is the number of particles inside $r, \ln \Lambda$ is the Coulomb logarithm. The ratio of the system lifetime $t_{0}$ to $\tau_{r}$ should be low enough to guarantee the negligibility of the relaxation. A real halo contains $\sim 10^{65}$ particles, hence the collisions are wholly immaterial. The quantity of test bodies in simulations is incomparably lower. The closer we approach the halo center, the smaller $N(r)$ and $r / v$ are, and the shorter is $\tau_{r}$. Thus the central region of the halos is the most problematic for the simulations: the profile inside some convergence radius $r_{\text {conv }}$ may be already corrupted by the collisions. It is commonly assumed that $r_{\text {conv }}$ is defined by a certain value of $t_{0} / \tau_{r}$.

The commonly-used criterion of the convergence of $N$-body simulations is the stability of the central density profile (Power et al. 2003). The simulations indeed show that the central NFWlike cusp is formed quite rapidly $\left(t<\tau_{r}\right)$ and then is stable and insensitive to the simulation parameters. However, the convergence criteria obtained with this method are surprisingly optimistic: the cusp is stable at least up to $t=1.7 \tau_{r}$ and probably much longer (Power et al. 2003). Hayashi et al. (2003) and Klypin et al. (2013) reported that the cusp is stable even at tens of relaxation times and smears out only at $t \sim 40 \tau_{r}$. The reasons why the collision influence is negligible at a time interval exceeding the relaxation time are not quite clear. Nevertheless, criterion $t=1.7 \tau_{r}$ (Power et al. 2003) is routinely used in modern simulations (Navarro et al. 2010).

The criteria based on the density profile stability have a weak point, however: the stability does not guarantee the absence of the collisional influence. Considerations based on the FokkerPlanck equation (Evans \& Collett 1997; Baushev 2013b) show that an NFW-like profile ( $\rho \propto r^{-\beta}, \beta \simeq 1$ ) is an attractor: the Fokker-Planck diffusion transforms any reasonable initial distribution into it in a time shorter than $\tau_{r}$, and then the cuspy profile should survive much longer than $\tau_{r}$, since the Fokker-Planck 
diffusion is self-compensated in this case. Therefore the cusp is stable and insensitive to the simulation parameters; at $t \sim 50 \tau_{r}$ it is destroyed by higher-order terms of the Boltzmann collision integral, disregarded by the Fokker-Planck approach (Quinlan 1996; Baushev 2013b). This scenario perfectly describes the behavior of real $N$-body simulations: the NFW-like cusp appears at $t<\tau_{r}$, remains stable up to tens of relaxation times, and then is smoothed. However, the shape of the cusp is in this case defined by the test particle collisions, that is, by a purely numerical effect. The only reliable criterion of negligibility of the unphysical collisions is $t \ll \tau_{r}$. This means that $r_{\text {conv }}$ is several times larger than predicted by the criterion $t=1.7 \tau_{r}$ (Power et al. 2003). Thus the criteria based on the profile stability are most likely too optimistic and underestimate the influence of numerical effects. The problem needs further investigation.

Contrary to the simulations, observations show a fairly smooth core in the centers of, at least, galaxy halos (de Blok et al. 2001; de Blok \& Bosma 2002; Marchesini et al. 2002; Gentile et al. 2007). Chemin et al. (2011) removed the baryon contribution and found that the dark matter distribution in the central regions of a large array of galaxies may well be fitted by the Einasto profile with a low index $(n \simeq 0.5)$ that corresponds to a cored profile. The central densities of the dwarf spheroidal satellites of the Andromeda galaxy are also low and favor the cored profiles (Tollerud et al. 2012), although the profiles in this case can be modified by the dynamical friction and tidal effects, since the satellites are situated inside the virial radius of the host galaxy. However, recent observations of dwarf spheroidal galaxies also indicate no cusps in their centers (Oh et al. 2011; Governato et al. 2012). This makes attempts to explain the soft cores of the central density profiles by the influence of the baryonic component dubious: the dwarfs contain only a very minor fraction of baryons.

Many galaxies (at least, the spiral ones) show quite an extensive region in their dark matter halo with a $\rho \propto r^{-2}$ profile: the region corresponds to a characteristic flat tail in their rotation curves. This feature allowed proving the existence of the dark matter by Rubin et al. (1978). Meanwhile, none of two profiles (Navarro-Frenk-White or Einasto) that are commonly used to fit the halos in the $N$-body simulations has such a region. Certainly, the current power-law index $\gamma=\mathrm{d} \log \rho / \mathrm{d} \log r$ of both the profiles reaches -2 at some point. However, the index changes continuously in both cases, the point where $\gamma=-2$ is marked not, therefore we cannot expect an extensive region with $\rho \sim r^{-2}$. Of course, the real structure formation is a much more complex process than the simulations, and the origin of the region could be a result of the influence of the baryon component, substructures, galaxy disk, etc. However, the persistence of the isothermal-like shape $\rho \sim r^{-2}$ in the density profiles of a vast collection of galaxies with very different physical properties (Sofue $\&$ Rubin 2001) suggests a more fundamental and more universal physical reason.

Finally, observations indicate that the multiplication of the halo central density $\rho_{\mathrm{c}}$ by the core radius $r_{\text {core }}$ is almost constant for a wide variety of galaxies, while their physical parameters, including $\rho_{\mathrm{c}}$ and $r_{\text {core }}$ apart, change in a rather extensive range. This effect was first discovered by Kormendy \& Freeman (2004) and then confirmed by several independent observations (see Salucci et al. 2007; Donato et al. 2009 and references therein). To be able to compare results obtained using different profile models, we define the core radius $r_{\text {core }}$ as the radius, at which

$$
\frac{\mathrm{d} \log \rho\left(r_{\text {core }}\right)}{\mathrm{d} \log r}=-1
$$

Donato et al. (2009) used the Burkert profile (Burkert 1995)

$$
\rho(r)=\frac{\rho_{\mathrm{c}} r_{\mathrm{b}}^{3}}{\left(r+r_{\mathrm{b}}\right)\left(r^{2}+r_{\mathrm{b}}^{2}\right)} .
$$

It is easy to see that $r_{\text {core }}=r_{\mathrm{b}} / 2$. Recently, Donato et al. (2009) found that $\log \left(\rho_{\mathrm{c}} r_{\mathrm{b}}\right)=2.15 \pm 0.2$ in units of $\log \left(M_{\odot} \mathrm{pc}^{-2}\right)$ on the basis of the co-added rotation curves of $\sim 1000$ spiral galaxies, the mass models of individual dwarf irregular and spiral galaxies of late and early types with high-quality mass profiles, and the galaxy-galaxy weak-lensing signals from a sample of spiral and elliptical galaxies. They also showed that the observed kinematics of Local Group dwarf spheroidal galaxies are consistent with this value as well. The result was obtained for galactic systems belonging to various Hubble types whose mass profiles have been determined by several independent methods.

The aim of this article is to show that all the abovementioned features (a cored central profile, an extended region with a $\rho \propto r^{-2}$ profile, and $\rho_{\mathrm{c}} r_{\text {core }} \simeq$ const. relationship) appear automatically, if we assume that the relaxation of the galactic halos during their formation was not violent. The violentrelaxation scenario, usually leading to a cuspy density profile, was first suggested by Lynden-Bell (1967) for stellar systems. The idea of it is that strong small-scale gravitational fields appear during the halo relaxation, and as a result all the particles completely forget their initial states. Recent $N$-body simulations (Diemand et al. 2005, 2007; Diemand \& Kuhlen 2008) showed however, that this assumption is probably incorrect, and a significant part of the particles and subhalos "remember" their initial specific energies $\epsilon=\frac{v^{2}}{2}+\phi$ : they change quite moderately.

There may be several theoretical reasons for the absence of the violent relaxation (Baushev 2014). For instance, the efficiency of the violent relaxation rapidly drops with the growth of the initial radius $r$ of the area under consideration from the center of the object. Even the original paper (Lynden-Bell 1967) reported that the outer regions of the stellar clusters remained unrelaxed. Meanwhile, a dark matter halo originates from a perturbation that was initially linear and, in contrast to the formed structures, had a low density contrast. Consequently, the main contribution to the halo mass was made by the layers with large $r$, since their volume $4 \pi r^{2} \mathrm{~d} r$ dominates. This circumstance impedes the relaxation. Moreover, a significant part of the dark matter gradually accretes onto the already formed halo, when the strong gravitational field inhomogeneities have already disappeared (Wang et al. 2012).

All these reasons allow us to assume that the violent relaxation does not occur, at least, in some types of halos. Hereafter we assume that the relaxation of low-mass halos $\left(M_{\text {vir }} \lesssim\right.$ $10^{12} M_{\odot}$ ), corresponding to galaxies, is not violent. We assume that the relaxation is moderate in the following sense:

1. The final total specific energy $\epsilon_{\mathrm{f}}$ of most of the particles differs from the initial ones $\epsilon_{\mathrm{i}}$ no more than by a factor $c_{\mathrm{vir}} / 5$

$$
\frac{\epsilon_{\mathrm{f}}}{\epsilon_{\mathrm{i}}} \leq \frac{c_{\mathrm{vir}}}{5}
$$

2. There can be particles that violate condition (3), but their total mass should be small with respect to the halo mass inside $r=\frac{2 R_{\mathrm{vir}}}{c_{\mathrm{vir}}}$

$$
M<\int_{0}^{2 R_{\text {vir }} / c_{\text {vir }}} \mathrm{d} M_{\text {halo }} .
$$

The reason for this limitation will be clear from the subsequent text. 
Here we used the NFW halo concentration $c_{\text {vir }}$. As we will see, the real density profile may significantly differ from the NFW one, if conditions (3)-(4) are true. However, we use $c_{\text {vir }}$ because of its popularity and in view of the fact that characteristic values of $c_{\text {vir }}$ for various types of astronomical objects are well known. Condition (3) is too strict for galaxy clusters, since their concentrations are low $\left(c_{\text {vir }} \sim 3-5\right)$. Indeed, even for rather a dense cluster $\left(c_{\text {vir }}=6\right)$ Eq. (3) would mean that the energies of almost all the particles change by no more than $20 \%$ during the relaxation. The real relaxation is most likely more intensive; perhaps, this is the reason why the galaxy clusters have profiles close to NFW (Okabe et al. 2010).

In contrast, conditions (3) and (4) seem quite soft for galactic halos. Concentration $c_{\text {vir }} \simeq 12-17$ even for the giant Milky Way galaxy and probably much higher for low-mass galaxies. Consequently, assumption (3) means that the energy of most of the particles changes no more than by a factor of 3 with respect to the initial value. This behavior looks quite natural for a collisionless system. Condition (4) is also weak. Indeed, a halo of typical galactic concentration $\left(c_{\mathrm{vir}} \sim 15-20\right)$ contains $20-25 \%$ of its mass inside $r=\frac{2 R_{\mathrm{vir}}}{c_{\mathrm{vir}}}$; this means that condition (4) reduces to the constraint that the fraction of the particles that changed their energy by more than a factor of $c_{\text {vir }} / 3 \simeq 3$ is smaller than a quarter of the halo mass.

Of course, there is always some dark matter that violates condition (3). For instance, the particles that were in the center of the halo at the very beginning of the collapse, when their velocities (as well as the velocities of other particles) were low. Their energies changed by much more than Eq. (3) during the collapse, even if there was no relaxation at all. Indeed, they remain in the halo center during the collapse, while the gravitational potential of this area deepens by approximately a factor $c_{\text {vir }}$ because of the crowding of the matter toward the center. However, the density of these "ancient habitants" of the halo center was comparable with the average DM density of the Universe at the moment of the halo collapse, that is, it was only by a factor $\sim 5$ higher than the present-day value. Meanwhile, the central density of the Milky Way is higher by a factor $\sim 3 \times 10^{5}$ than the Universe DM density. Clearly, the "ancient habitants" do yield some density into the DM content of the Galactic center, but the contribution is negligible $\left(\sim 10^{-5}\right)$.

To conclude the introductory section, we should emphasize that the moderate relaxation is now no more than a hypothesis. However, as we will see, it leads to quite correct predictions of the central density profiles of galaxies.

In Sect. 2 we discuss the energy evolution of a collapsing dark matter halo and show that the distribution of the formed halo probably has a peculiar form. In Sect. 3 we calculate the density profile corresponding to this distribution. In Sect. 4 we discuss the obtained profile and compare it with observational data. Finally, in Sect. 4, we briefly summarize our results and discuss further implications.

\section{Energy distribution}

The assumption of the moderate energy evolution immediately leads to some important consequences. Hereafter we accept for simplicity that the halo is spherically symmetric. The presentday dark matter halos were formed from some primordial perturbations that existed in the early Universe. Initially, the perturbations grew linearly, but then they reached the nonlinear regime and collapsed. We consider the initial (at the moment when $\delta \rho / \rho \simeq 1$ ) energy distribution of the particles that later

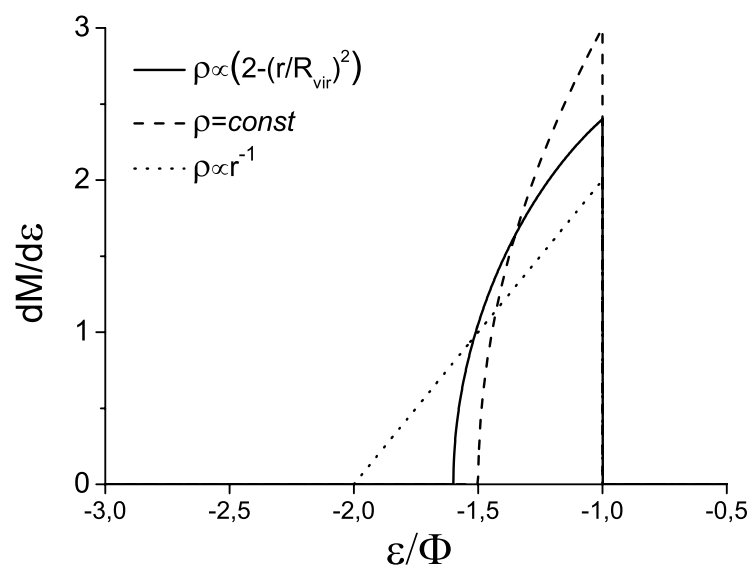

Fig. 1. Initial energy spectra $\frac{\mathrm{d} M}{\mathrm{~d} \epsilon}$ for three shapes of initial perturbation: $\rho \propto\left(2-\left(\frac{r}{R_{\text {vir }}}\right)^{2}\right)$ (solid line), $\rho=$ const. (dashed line), $\rho \propto r^{-1}$ (dotted line). In all the cases the spectra are similar, narrow, and strongly concentrated toward $\epsilon=-\Phi$.

formed the halo. It is determined by the gravitational field of the initial perturbation, and the closer a particle was to the center, the lower was its energy. However, the potential well of the initial perturbation cannot be deep, and the particles cannot have a very low energy, because thy are lumped in quite a narrow energy interval. Indeed, a trivial Newtonian calculation gives us the initial energy spectrum for various shapes of initial perturbations (Baushev 2014). We assume for simplicity that the size of the perturbation at the moment of the collapse is equal to the virial radius of the formed halo $R_{\text {vir }}$ : these values should be similar in the very general case (Gorbunov \& Rubakov 2010). We introduce the virial potential of the halo $\Phi=G \frac{M_{\mathrm{vir}}}{R_{\mathrm{vir}}}$. As an example, we consider the case when the initial density distribution of the perturbation has the shape $\rho \sim r^{-1}$ inside $R_{\mathrm{vir}}$. Then $M(r)=\int 4 \pi r^{2} \rho \mathrm{d} r=M_{\mathrm{vir}}\left(r / R_{\mathrm{vir}}\right)^{2}$ and

$\frac{\mathrm{d} \phi(r)}{\mathrm{d} r}=G \frac{M(r)}{r^{2}}=G \frac{M_{\mathrm{vir}}}{R_{\mathrm{vir}}^{2}} \quad \phi(r)=-\Phi\left(2-\frac{r}{R_{\mathrm{vir}}}\right)$.

Here we took into account that $\phi\left(R_{\text {vir }}\right)=-\Phi$. It follows from the general cosmological consideration that the initial velocity of the matter may be thought to be zero without loss of generality (Gorbunov \& Rubakov 2010). Therefore, the specific total energy of a particle is equal to the specific gravitational energy, that is, to the gravitational potential $\epsilon=\phi(r)$. By dividing $\mathrm{d} M(r)=\frac{2 r M_{\mathrm{vir}}}{R_{\mathrm{vir}}^{2}} \mathrm{~d} r$ by $\mathrm{d} \epsilon=\mathrm{d} \phi(r)=G \frac{M_{\mathrm{vir}}}{R_{\mathrm{vir}}^{2}} \mathrm{~d} r$, we obtain

$\frac{\mathrm{d} M}{\mathrm{~d} \epsilon}=\frac{\mathrm{d} M}{\mathrm{~d} \phi(r)}=\frac{2 r}{G}=\frac{2 M_{\mathrm{vir}}}{\Phi}\left(2+\frac{\epsilon}{\Phi}\right)$.

In a similar manner, we can obtain the initial energy spectra for various forms of initial perturbations (see (Baushev 2014) for details). Distributions $\frac{\mathrm{d} M}{\mathrm{~d} \epsilon}$ for three different shapes $(\rho \propto(2-$ $\left(\frac{r}{R_{\text {vir }}}\right)^{2}$ ) (solid line), $\rho=$ const. (dash line), $\rho \propto r^{-1}$ (dotted line)) are represented in Fig. 1. In all the cases the spectra are quite similar, narrow, and strongly concentrated toward $\epsilon=-\Phi$. Even an unphysically steep initial perturbation $\rho \sim r^{-1}$ contains only particles with $\epsilon \in[-2 \Phi ;-\Phi]$.

We now consider the formed halo. Its gravitational field is stationary. The motion of a particle in the central gravitational field $\phi(r)$ can be explicitly characterized by two integrals of motion: its specific angular momentum $\mu \equiv|[\boldsymbol{v} \times \boldsymbol{r}]|$ and specific 
energy $\epsilon=\frac{v_{r}^{2}}{2}+\frac{\mu^{2}}{2 r^{2}}+\phi$. Instead of $\epsilon$, it is more convenient to use the apocenter distance of the particle $r_{0}$, which is the largest distance that the particle can move away from the center. It is bound with $\epsilon$ as $\epsilon=\phi\left(r_{0}\right)+\mu^{2} /\left(2 r_{0}^{2}\right)$. We may introduce distribution function $f\left(r_{0}\right)$ of the particles of the formed halo over $r_{0}$

$\mathrm{d} m=f\left(r_{0}\right) \mathrm{d} r_{0} \quad \int_{0}^{R_{\mathrm{vir}}} f\left(r_{0}\right) \mathrm{d} r_{0}=M_{\text {vir }}$.

As we will see, if conditions (3)-(4) are satisfied, $f\left(r_{0}\right)$ has a very peculiar appearance.

It is extremely important for our consideration that the potential well of the collapsed halo is much deeper then the initial one. The depth (i.e., the value of $\phi(0))$ depends on the halo profile: for the NFW $\phi(r) \simeq-c_{\text {vir }} \Phi$, if $c_{\text {vir }} \gg 1$ (Baushev 2012). Although density profiles of real galaxies are much more complex, $\phi(r) \simeq-c_{\mathrm{vir}} \Phi$ may still be a good approximation. For instance, we may accept for the Milky Way $M_{\mathrm{vir}}=10^{12} M_{\odot}$, $R_{\mathrm{vir}}=250 \mathrm{kpc}, c_{\mathrm{vir}} \simeq 15$ (Klypin et al. 2002): then $\Phi \simeq$ $(130 \mathrm{~km} \mathrm{~s})^{-2}$. Meanwhile, the Galaxy escape speed near the solar system unambiguously exceeds $525 \mathrm{~km} \mathrm{~s}^{-1}$ (Carney \& Latham 1987) and may in principle be much higher $\left(650 \mathrm{~km} \mathrm{~s}^{-1}\right.$ or even higher (Marochnik \& Suchkov 1984; Binney \& Tremaine 2008)). Accordingly, $|\phi(0)| \gtrsim c_{\text {vir }}\left|\phi\left(R_{\text {vir }}\right)\right|=c_{\text {vir }} \Phi$.

As we showed, initial energy spectra are very similar for any reasonable shape of the initial perturbation. We consider for the sake of definiteness an initial perturbation $\rho \propto\left(2-\left(\frac{r}{R_{\mathrm{vir}}}\right)^{2}\right)$, because it seems to be a good approximation for a real one. We can see in Fig. 1 that most the particles have $\epsilon \simeq-\Phi$, and there are no particles with $\epsilon<-1.6 \Phi$. Consequently, the particles obeying condition (3) may not have $\epsilon<-\frac{c_{\text {vir }}}{3} 1.6 \Phi \simeq-\frac{c_{\text {vir }}}{2} \Phi$, and even the fraction of the particles with $\epsilon \simeq-\frac{c_{\mathrm{vi}}}{2} \Phi$ is small: the initial spectrum contains only a few particles with $\epsilon \simeq-1.6 \Phi$. We estimate $r_{0}$, corresponding to a particle with $\epsilon=-1.6 \Phi$. Of course, it depends on the density profile of the halo and on the particle angular momentum. The influence of the latter factor can be easily taken into account: a nonzero angular momentum decreases $r_{0}$ of a particle of a given energy $\epsilon$, but cannot decrease it by a factor exceeding 2. Indeed, a particle of given energy $\epsilon$ in a given central gravitational field has the largest $r_{0}$ if $\mu=0$, and its orbit is radial. The ratio of $r_{0}$ for the radial and the circular cases is 2 in the instance of the gravitational field of a point mass. It is easy to see that the ratio can only be lower, if we consider a distributed density profile instead of a point mass: if the dark matter is spread, the more compact circular orbit encloses a smaller central mass than in the point-mass case. Moreover, $\mathrm{N}$-body simulations suggest that the orbits of most of the particles are elongated (Hansen et al. 2006). In this case the influence of the angular momentum on $r_{0}$ is negligible.

A particle of energy $\epsilon \simeq-\frac{c_{\mathrm{vir}}}{2} \Phi$ has $r_{0} \simeq \frac{2 R_{\mathrm{vir}}}{c_{\mathrm{vir}}}$ in the case of an NFW profile. The potential wells of real galaxies are most likely deeper than the best-fit NFW predicts (probably because of the influence of the much more concentrated baryon component). For instance, the escape velocity from the center of an NFW halo with $M_{\text {vir }}=10^{12} M_{\odot}$ and $c_{\text {vir }} \simeq 15$ (as we could see, these values approximately correspond to the dark matter halo of the Milky Way) is $\simeq 300 \mathrm{~km} \mathrm{~s}^{-1}$, while the real escape speed from the center of the Galaxy is at least twice as high (Carney \& Latham 1987). The deeper the potential well, the larger is $r_{0}$ that corresponds to the same $\epsilon$; consequently, $r_{0}=\frac{2 R_{\mathrm{vir}}}{c_{\mathrm{vir}}}$ is a conservative estimate of $r_{0}$ of a particle with energy $\epsilon \simeq-\frac{c_{\text {vir }}}{2} \Phi$. Consequently, particles obeying condition (3) cannot have smaller $r_{0}$. However, the halo contains a significant part of its mass $(\sim 25 \%)$ inside $r=\frac{2 R_{\mathrm{vir}}}{c_{\mathrm{vir}}}$.

A114, page 4 of 8
The absence of violent relaxation leads to a very important consequence: the density profile in the center of the halo is formed by the particles that arrive from the outside. Since their $r_{0}$ are larger than $\frac{2 R_{\mathrm{vir}}}{c_{\mathrm{vir}}}$, some part of their trajectories lie outside of this area. Of course, the real situation is more complex, and there are always particles that violate condition (3). However, condition (4) guarantees that their contribution inside $r=\frac{2 R_{\mathrm{vir}}}{c_{\mathrm{vir}}}$ is small. As we could see, condition (4) is quite soft for the real systems.

We can also expect that most of the particles have $r_{0} \sim R_{\mathrm{vir}}$. Indeed, the initial energies of the particles were very close to the virial one $\Phi=-G \frac{M_{\mathrm{vir}}}{R_{\mathrm{vir}}}$. Since the total energy of the system is conserved, the average energy of the particles remain close to $-\Phi$; consequently, all the particles may not drop their energies by a factor $\sim c_{\text {vir }} / 5$. The particle energy exchange is a more or less random process, and we may expect that the particle energy near the average value $-\Phi$ is much more probable than the minimum possible $-\frac{c_{\text {vir }}}{5} \Phi$. Consequently, even the fraction of particles with $r_{0} \simeq \frac{2 R_{\mathrm{vir}}}{c_{\mathrm{vir}}}$ should be small. However, this statement is less rigid (and less important for us) than the above-mentioned dominance of the particles with $r_{0} \gg r$ in the center.

A region with a dominant fraction of particles with $r_{0} \gg r$ inevitably occurs in the center of the halo, if the relaxation is moderate. It even appears if the relaxation is much more violent than Eq. (3) (for instance, if $\epsilon_{\mathrm{f}} / \epsilon_{\mathrm{i}} \sim c_{\mathrm{vir}} / 2$ ): the lowest energy of the particles is still higher than $\phi(0) \simeq-c_{\text {vir }} \Phi$ in this case. However, the radius of the area is then much smaller. Hereafter we use condition (3), and the area is quite large in this case: $\frac{2 R_{\mathrm{vir}}}{c_{\mathrm{vir}}} \simeq 30 \mathrm{kpc}$ for the Milky Way galaxy.

\section{Calculations}

The density distribution in the center of the halo, created by the particles that arrive in this region from the outside, is universal and insensitive to the shape of distribution $f\left(r_{0}\right)$ (Baushev 2014, 2013a). First of all, we specify the angular momentum distribution of the particles. According to results of the numerical simulations (see, for instance, Kuhlen et al. 2010), the distribution over $v_{\tau}$ deviates, but is still similar to Gaussian. We assume for simplicity that their specific angular momentum has a Gaussian distribution

$\mathrm{d} m \propto \frac{2 \mu}{\alpha^{2}} \exp \left(-\frac{\mu^{2}}{\alpha^{2}}\right) \mathrm{d} \mu$,

where $\alpha \equiv \alpha\left(r_{0}\right)$ is the width of the distribution, depending on $r_{0}$. The total distribution can be rewritten as

$\mathrm{d} m=f\left(r_{0}\right) \frac{2 \mu}{\alpha^{2}} \exp \left(-\frac{\mu^{2}}{\alpha^{2}}\right) \mathrm{d} r_{0} \mathrm{~d} \mu$.

As we have already mentioned, most of the particles have $r_{0} \sim R_{\mathrm{vir}}$, and therefore only those with small $\mu$ can penetrate into the area of our interest $r \sim r_{\text {core }} \ll R_{\text {vir }}$. This means that the distribution in the halo center is mainly determined by the behavior of Eq. (9) at $\mu \simeq 0$, where Eq. (9) is finite. Thus our calculation is not sensitive to the distribution over $\mu$ : we would obtain a very similar result for any other distribution, which has the same value $2 \mu f\left(r_{0}\right) / \alpha^{2}\left(r_{0}\right)$ when $\mu \rightarrow 0$.

Since the particle energy conserves

$\frac{\mu^{2}}{2 r_{0}^{2}}+\phi\left(r_{0}\right)=\epsilon=\frac{v_{r}^{2}}{2}+\frac{\mu^{2}}{2 r^{2}}+\phi(r)$, 
the radial and tangential components of the particle velocity are equal to

$v_{r}=\sqrt{2\left(\phi\left(r_{0}\right)-\phi(r)\right)-\mu^{2}\left(\frac{1}{r^{2}}-\frac{1}{r_{0}^{2}}\right)} \quad v_{\tau}=\frac{\mu}{r}$.

The zero of the radicand gives us the maximum angular momentum of a particle with which it can reach radius $r$,

$\mu_{\max }^{2}=2\left(\phi\left(r_{0}\right)-\phi(r)\right)\left(\frac{1}{r^{2}}-\frac{1}{r_{0}^{2}}\right)^{-1}$.

We may rewrite Eq. (11) as

$v_{r}=\frac{\sqrt{r_{0}^{2}-r^{2}}}{r r_{0}} \sqrt{\mu_{\max }^{2}-\mu^{2}}$.

We also need the half-period of the particle, that is, the time it takes for the particle to fall from its largest to the smallest radius,

$T\left(r_{0}, \mu\right)=\int_{r_{\min }}^{r_{0}} \frac{\mathrm{d} r}{v_{r}}$.

$T$ is, generally speaking, a function of $r_{0}$ and $\mu$. However, as was shown in Baushev (2014), for the particles that can reach the cental region the dependence on $\mu$ is extremely weak (the reason is that the function $T\left(r_{0}, \mu\right)$ slowly changes near the extremum at $\mu=0)$. Therefore we may approximate $T\left(r_{0}, \mu\right) \simeq T\left(r_{0}, 0\right) \equiv$ $T\left(r_{0}\right)$.

A particle of mass $m$ contributes to the halo density throughout the interval between $r_{0}$ and the smallest radius the particle can reach. The contribution in an interval $\mathrm{d} r$ is proportional to the time the particle spends in this interval (Baushev 2011)

$\frac{\mathrm{d} m}{m}=\frac{\delta \rho \cdot 4 \pi r^{2} \mathrm{~d} r}{m}=\frac{\mathrm{d} t}{T\left(r_{0}\right)}=\frac{\mathrm{d} r}{v_{r} T\left(r_{0}\right)}$.

Here $\delta \rho$ is the contribution of the particle to the total halo density at radius $r$. We obtain that $\delta \rho=\frac{m}{4 \pi r^{2} v_{r} T\left(r_{0}\right)}$. To determine the total halo density, we substitute here a mass element (9) instead of $m$ and integrate over $\mathrm{d} r_{0}$ and $\mathrm{d} \mu$,

$\rho=\int_{0}^{R_{\text {vir }}} \frac{f\left(r_{0}\right) \mathrm{d} r_{0}}{4 \pi r^{2} T\left(r_{0}\right) \alpha^{2}\left(r_{0}\right)} \int_{0}^{\mu_{\max }} \frac{2 \mu}{v_{r}} \exp \left(-\frac{\mu^{2}}{\alpha^{2}\left(r_{0}\right)}\right) \mathrm{d} \mu$.

If we substitute Eq. (13) for $v_{r}$, the second integral can be taken analytically:

$\int_{0}^{\mu_{\max }} \frac{2 \mu}{v_{r}} \exp \left(-\frac{\mu^{2}}{\alpha^{2}\left(r_{0}\right)}\right) \mathrm{d} \mu=\frac{2 r r_{0} \alpha\left(r_{0}\right)}{\sqrt{r_{0}^{2}-r^{2}}} D\left(\frac{\mu_{\max }}{\alpha\left(r_{0}\right)}\right)$,

where $D(x) \equiv \mathrm{e}^{-x^{2}} \int_{0}^{x} \mathrm{e}^{t^{2}} \mathrm{~d} t$ is the Dawson function. Since we calculate the density profile of the central region and use assumption $r \ll r_{0}$, we can significantly simplify Eq. (17). In particular, it follows from Eq. (12) that $\mu_{\max } \simeq r \sqrt{2\left(\phi\left(r_{0}\right)-\phi(0)\right)}$. We obtain

$\rho=\int_{0}^{\infty} \frac{f\left(r_{0}\right)}{2 \pi \alpha\left(r_{0}\right) T\left(r_{0}\right) r} D\left(r \frac{\sqrt{2\left(\phi\left(r_{0}\right)-\phi(0)\right)}}{\alpha\left(r_{0}\right)}\right) \mathrm{d} r_{0}$.

We can factor out the Dawson function from the integral using the above-mentioned properties of function $f\left(r_{0}\right)$ (see the end

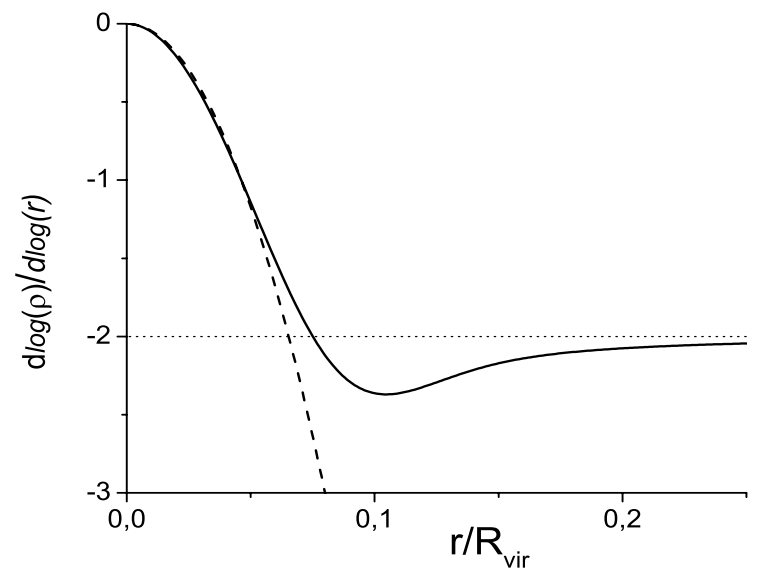

Fig. 2. Density profile of the model under consideration (20) (with $r_{\mathrm{c}}=$ $0.05 R_{\mathrm{vir}}$, solid line). An Einasto profile with $n=0.5$ and $r_{\mathrm{s}}=0.017 R_{\mathrm{vir}}$ is plotted for comparison (dashed line).

of the Energy distribution section). First, it is almost equal to zero for small $r_{0}$ : this means that the integration in Eq. (18) is performed not from 0 , but from $\frac{2 R_{\mathrm{vir}}}{c_{\mathrm{vir}}}$. Second, as we could see, the formed halo is dominated by the particles with $r_{0} \sim$ $R_{\text {vir. }}$. It follows that the main contribution to the integral in Eq. (18) is given by the part close to the upper limit $r_{0} \simeq R_{\mathrm{vir}}$ : roughly speaking, by $r_{0} \in\left[R_{\mathrm{vir}} / 2 ; R_{\mathrm{vir}}\right]$. These two properties of $f\left(r_{0}\right)$ mean that $f\left(r_{0}\right)$ sharply depends on $r_{0}$ at this interval. Conversely, $\alpha\left(r_{0}\right)$ probably does not change much in interval $\left[R_{\mathrm{vir}} / 2 ; R_{\mathrm{vir}}\right]: \alpha\left(r_{0}\right)$ is widely believed to be a power-law dependence with the index between -1 and 1 (Hansen et al. 2006). $\sqrt{2\left(\phi\left(r_{0}\right)-\phi(0)\right)}$ changes even more slowly: for instance, $\sqrt{\left(\phi\left(R_{\mathrm{vir}}\right)-\phi(0)\right) /\left(\phi\left(R_{\mathrm{vir}} / 2\right)-\phi(0)\right)} \simeq 1.13$ for the NFW profile with $c_{\text {vir }}=15$. Moreover, $D$ is a finite and not very sharp function of its argument. Comparing this with the sharp behavior of $f\left(r_{0}\right)$, we may neglect the weak dependence of the argument of function $D$ in Eq. (18) on $r_{0}$ and substitute some value, averaged over the halo (see the Appendix for details),

$r_{\mathrm{c}}=\left\langle\frac{\alpha\left(r_{0}\right)}{\sqrt{2\left(\phi\left(r_{0}\right)-\phi(0)\right)}}\right\rangle \simeq \frac{\left\langle\alpha\left(r_{0}\right)\right\rangle}{\sqrt{2|\phi(0)|}}$.

Then we can rewrite Eq. (18) and obtain the final result:

$\rho=\rho_{\mathrm{c}} \frac{r_{\mathrm{c}}}{r} D\left(\frac{r}{r_{\mathrm{c}}}\right), \quad \rho_{\mathrm{c}}=\frac{1}{2 \pi r_{\mathrm{c}}} \int_{0}^{\infty} \frac{f\left(r_{0}\right) \mathrm{d} r_{0}}{\alpha\left(r_{0}\right) T\left(r_{0}\right)}$.

Since $D\left(r / r_{\mathrm{c}}\right) \simeq r / r_{\mathrm{c}}$, when $r / r_{\mathrm{c}} \rightarrow 0, \rho_{\mathrm{c}}$ is really the central density of the halo. As we can see, it is always finite. At the same time, the shape of the density profile only depends on parameter $r_{\mathrm{c}}$.

\section{Discussion}

Figure 2 represents profile (20) with $r_{\mathrm{c}}=0.05 R_{\mathrm{vir}}$ (solid line). An Einasto profile with $n=0.5$ and $r_{\mathrm{s}}=0.017 R_{\mathrm{vir}}$ is plotted for comparison (dashed line). The model profile is very similar to the Einasto profile in the center; consequently, it fits experimental data well (Chemin et al. 2011). The second consequence is that Eq. (20) describes a cored profile with $r_{\text {core }} \simeq r_{\mathrm{c}}$; using criterion (1), we obtain $r_{\text {core }} \simeq 0.924 r_{\mathrm{c}}$.

Profile (20) in all conditions transforms into $\rho \propto r^{-2}$ at large distances, which may explain the persistence of the flat regions in the rotation curves of a vast collection of galaxies with very 
different physical properties. However, a question arises: we assume that $r_{0} \gg r$ during the derivation of Eq. (20). Is this approximation (and, consequently, Eq. (20)) still valid for large $r$, where the profile behaves as $\rho \propto r^{-2}$ ? As we showed, Eq. (20) is valid for $r<\frac{2 R_{\mathrm{vir}}}{c_{\mathrm{vir}}}$. Meanwhile, the $\rho \propto r^{-2}$ profile appears if $r \gg r_{\mathrm{c}}$. The value of $r_{\mathrm{c}}$ depends on $\langle\alpha\rangle$ and $\sqrt{2|\phi(0)|}$ according to Eq. (19). We show below that the best agreement between the theory and observations is achieved if $\langle\alpha\rangle$ is respectively small (32). Substituting Eq. (32) and $|\phi(0)| \simeq c_{\text {vir }} \Phi$ into (19), we can roughly estimate $r_{\mathrm{c}} \simeq R_{\mathrm{vir}} /\left(28 \sqrt{2 c_{\mathrm{vir}}}\right)$. We illustrate this on the example of the Milky Way galaxy. Here $\frac{2 R_{\mathrm{vir}}}{c_{\mathrm{vir}}} \simeq 30 \mathrm{kpc}$, that is, approximately the disk radius; $r_{\mathrm{c}} \simeq R_{\mathrm{vir}} /\left(28 \sqrt{2 c_{\mathrm{vir}}}\right) \simeq$ $1.6 \mathrm{kpc}$, which is comparable with the bulge size. So $\frac{2 R_{\mathrm{vir}}}{c_{\mathrm{vir}}} \gg r_{\mathrm{c}}$, Eq. (20) is still valid for $r \gg r_{\mathrm{c}}$, and we may expect an extended region with $\rho \propto r^{-2}$ between $\sim 2 r_{\mathrm{c}} \simeq 3.2$ and $\sim 30 \mathrm{kpc}$.

Now we can investigate how the multiplication $\rho_{\mathrm{c}} r_{\mathrm{c}}$ depends on the halo mass in our model. According to Eq. (20),

$\rho_{\mathrm{c}} r_{\mathrm{c}}=\frac{1}{2 \pi} \int_{0}^{\infty} \frac{f\left(r_{0}\right) \mathrm{d} r_{0}}{\alpha\left(r_{0}\right) T\left(r_{0}\right)}$.

We can significantly simplify this equation with the help of the same technic that we used to transform Eqs. (18) into (20) (see Appendix): neglect the fairly weak dependencies of functions $\alpha\left(r_{0}\right)$ and $T\left(r_{0}\right)$ on $r_{0}$ (compared with $f\left(r_{0}\right)$ ), and substitute some values, averaged over the halo. Then Eq. (20) may be rewritten as

$\rho_{\mathrm{c}} r_{\mathrm{c}} \simeq \frac{1}{2 \pi} \frac{\int_{0}^{\infty} f\left(r_{0}\right) \mathrm{d} r_{0}}{\langle\alpha\rangle\langle T\rangle}=\frac{1}{2 \pi} \frac{M_{\mathrm{vir}}}{\langle\alpha\rangle\langle T\rangle}$.

Now we estimate $\langle\alpha\rangle$ and $\langle T\rangle$. To begin with, we assume that $\langle\alpha\rangle$ has the highest possible value: it can hardly be higher than

$\langle\alpha\rangle=\frac{1}{4} \sqrt{G M_{\mathrm{vir}} R_{\mathrm{vir}}}$

because a significant fraction of the halo particles would not be gravitationally bound in the opposite case. Below we discuss the applicability of this assumption.

The half-period $T\left(r_{0}\right)$ is mainly determined by the gravitational acceleration at $r_{0}$ (where $v_{r}=0$ ) and is not very sensitive to the density distribution in the halo center. As we showed, a significant part of the particles should have $r_{0} \sim R_{\mathrm{vir}}$. Therefore we accept as an estimate of $\langle T\rangle$ the time necessary for a particle with no angular momentum to fall from $r=R_{\mathrm{vir}} / 2$ on a point mass,

$\langle T\rangle=\int_{0}^{R_{\mathrm{vir}}} \frac{\mathrm{d} r}{v_{r}}=\frac{\pi}{8} \frac{R_{\mathrm{vir}}^{3 / 2}}{\sqrt{G M_{\mathrm{vir}}}}$.

Indeed, $\left\langle r_{0}\right\rangle$ can hardly be smaller than $R_{\text {vir }} / 2$ : as we showed, $r_{0} \sim R_{\text {vir }}$ for most of the particles in our model. On the other hand, we underestimate $\langle T\rangle$ by considering a point mass instead of the real distribution. Consequently, Eq. (24) most likely underestimates $\langle T\rangle$.

It is convenient to introduce $\varrho$ - the average halo density: $M_{\mathrm{vir}}=\frac{4}{3} \pi R_{\mathrm{vir}}^{3} \varrho$. Substituting Eqs. (23) and (24) to (22), we obtain

$\rho_{\mathrm{c}} r_{\mathrm{c}}=\frac{2^{16 / 3}}{\pi^{4 / 3} 3^{2 / 3}} M_{\mathrm{vir}}^{1 / 3} \varrho^{2 / 3} \simeq 4.21 M_{\mathrm{vir}}^{1 / 3} \varrho^{2 / 3}$.

As $M_{\text {vir }}$ grows, multiplier $M_{\text {vir }}^{1 / 3}$ slowly increases, while $\varrho^{2 / 3}$ slowly decreases, since smaller halos formed at higher $z$, when the Universe density was higher. Roughly speaking, the density of a structure is proportional to the density of the Universe at the moment $z$ when it collapsed (Cooray \& Sheth 2002), that is, $\varrho \propto(z+1)^{3}$. Indeed, structures form when their density contrast $\delta \rho / \rho$ reaches a certain value (close to 1 ) that does not depend on the mass (Gorbunov \& Rubakov 2010, Sect. 7.2.2). Strictly speaking, the dependence of $z$ on $M_{\mathrm{vir}}$ is ambiguous: the initial perturbations can be considered as a random Gaussian field, and structures of the same mass can collapse at different $z$. Nevertheless, we may consider an averaged redshift $z$ of the collapse of structures of mass $M_{\mathrm{vir}}$.

We have accepted for the Milky Way $M_{\mathrm{vir}}=M_{\mathrm{MW}}=$ $10^{12} M_{\odot}, R_{\text {vir }}=250 \mathrm{kpc}$ (Klypin et al. 2002). It corresponds to $\varrho_{\mathrm{MW}}=1.5 \cdot 10^{4} M_{\odot} \mathrm{kpc}^{-3}$. Instead of $M_{\mathrm{vir}}$, we can characterize the structures by the present-day wave number $k$ of the primordial perturbations from which they were formed. Of course, $M_{\mathrm{vir}} \propto k^{-3}$. The Milky Way mass $M_{\mathrm{vir}} \simeq 10^{12} M_{\odot}$ corresponds to $k_{\mathrm{MW}} \simeq(0.6 \mathrm{Mpc})^{-1}$ (Gorbunov \& Rubakov 2010). So $k=k_{\mathrm{MW}}\left(M_{\mathrm{vir}} / M_{\mathrm{MW}}\right)^{-1 / 3}$.

The shape of dependence $z\left(M_{\mathrm{vir}}\right)$ is defined by the cosmological model. We consider the very standard $\Lambda$ CDM scenario with $H_{0}=67.3\left(\mathrm{Mpc}^{-1} \mathrm{~km} \mathrm{~s}^{-1}\right)$ (i.e., $H_{0}^{-1}=4.58 \times 10^{17} \mathrm{~s}$ ), $\Omega_{\mathrm{m}}=0.315$ (Planck Collaboration XVI 2014). In this case, $z$ logarithmically depends on $M_{\mathrm{vir}}$ (Gorbunov \& Rubakov 2010, Eq. (5.47))

$z+1 \propto \ln \left(\frac{0.4(\sqrt{2}-1) c}{H_{0} \sqrt{\Omega_{\mathrm{m}}} \sqrt{z_{\mathrm{eq}}+1}} k\right)$.

We accept $z_{\text {eq }}=3100$ for the equi-density redshift of radiation and matter (Gorbunov \& Rubakov 2010). Substituting here $\varrho \propto$ $(z+1)^{3}$ and $k=k_{\mathrm{MW}}\left(M_{\mathrm{vir}} / M_{\mathrm{MW}}\right)^{-1 / 3}$, we obtain

$\varrho \propto \ln ^{3}\left[\frac{0.4(\sqrt{2}-1) c k_{\mathrm{MW}}}{H_{0} \sqrt{\Omega_{\mathrm{m}}} \sqrt{z_{\mathrm{eq}}+1}}\left(\frac{M_{\mathrm{vir}}}{M_{\mathrm{MW}}}\right)^{-1 / 3}\right]$.

It is convenient to introduce

$X \equiv \frac{0.4(\sqrt{2}-1) c k_{\mathrm{MW}}}{H_{0} \sqrt{\Omega_{\mathrm{m}}} \sqrt{z_{\mathrm{eq}}+1}}$.

Then Eq. (27) may be rewritten as

$\varrho \propto \ln ^{3}\left[X\left(M_{\mathrm{vir}} / M_{\mathrm{MW}}\right)^{-1 / 3}\right] \propto\left(1-\frac{\ln \left(M_{\mathrm{vir}} / M_{\mathrm{MW}}\right)}{3 \ln X}\right)^{3}$.

Consequently,

$\varrho=\varrho_{\mathrm{MW}}\left(1-\frac{\ln \left(M_{\mathrm{vir}} / M_{\mathrm{MW}}\right)}{3 \ln X}\right)^{3}$.

Now we can insert this value into Eq. (25). To compare the result with observations, we need to calculate $\rho_{\mathrm{c}} r_{\mathrm{b}}$ instead of $\rho_{\mathrm{c}} r_{\mathrm{c}}$. By using a profile-independent definition of the core radius (1), we obtain $r_{\text {core }}=r_{\mathrm{b}} / 2$ and $r_{\text {core }} \simeq 0.924 r_{\mathrm{c}}$. Consequently, $\rho_{\mathrm{c}} r_{\mathrm{c}} \simeq$ $0.541 \rho_{\mathrm{c}} r_{\mathrm{b}}$, and we derive the final result

$\rho_{\mathrm{c}} r_{\mathrm{b}}=7.78 \varrho_{\mathrm{MW}}^{2 / 3} M_{\mathrm{MW}}^{1 / 3}\left(\frac{M_{\mathrm{vir}}}{M_{\mathrm{MW}}}\right)^{1 / 3}\left(1-\frac{\ln \left(M_{\mathrm{vir}} / M_{\mathrm{MW}}\right)}{3 \ln X}\right)^{2}$.

Observations suggest that $\log \left(\rho_{\mathrm{c}} r_{\mathrm{b}}\right) \simeq$ const. $=2.15 \pm 0.2$ in units of $\log \left(M_{\odot} \mathrm{pc}^{-2}\right)$ for a large array of elliptic and spiral galaxies, and probably for the Local Group dwarf spheroidal galaxies (Donato et al. 2009). Figure 3 represents the dependence (25) (solid line) predicted by the moderate relaxation model. Clearly, 


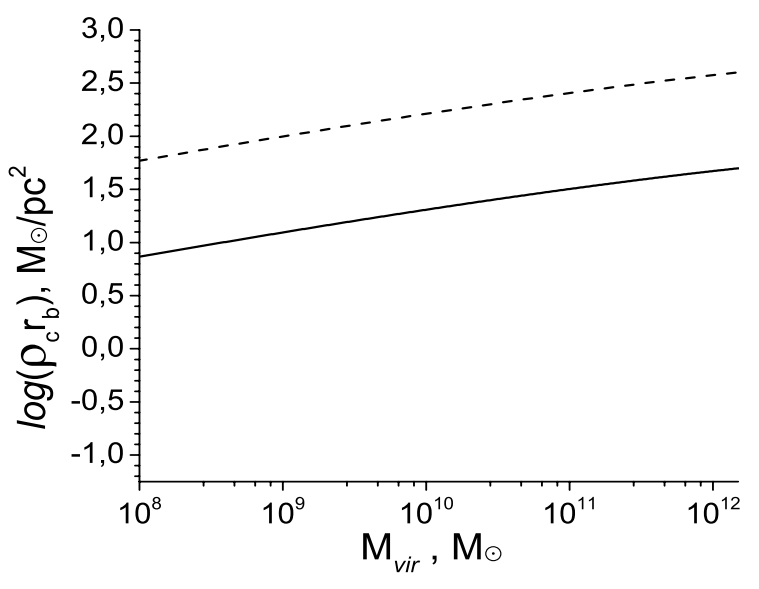

Fig. 3. Value (31) of $\rho_{\mathrm{c}} r_{\mathrm{b}}$ for various halo masses (solid line). For the entire range of galactic masses $\left(M_{\mathrm{vir}} \simeq 10^{9}-10^{12} M_{\odot}\right)$ multiplication $\rho_{\mathrm{c}} r_{\mathrm{b}}$ remains almost constant. According to Eq. (22), $\rho_{\mathrm{c}} r_{\mathrm{b}}$ is inversely proportional to $\langle\alpha\rangle$. The dashed line represents $\rho_{\mathrm{c}} r_{\mathrm{b}}$, if we use a lower value of $\langle\alpha\rangle$ (Eq. (32) instead of (23)). This agrees well with observations $\left(\log \left(\rho_{\mathrm{c}} r_{\mathrm{b}}\right)=2.15 \pm 0.2\right.$ in units of $\left.\log \left(M_{\odot} \mathrm{pc}^{-2}\right)\right)$.

the multiplication $\rho_{\mathrm{c}} r_{\mathrm{b}}$ is not perfectly constant; however, it changes only by a factor of three when the virial mass of galaxies varies from $10^{9} M_{\odot}$ to $10^{12} M_{\odot}$, which covers almost the entire galaxy mass range. This means that the variation of $\rho_{\mathrm{c}} r_{\mathrm{b}}$ does not exceed the $3 \sigma$ interval of the observations, and from this point of view, $\rho_{\mathrm{c}} r_{\mathrm{b}}$ may be considered as constant.

Thus, the moderate evolution model naturally predicts an almost constant multiplication $\rho_{\mathrm{c}} r_{\mathrm{b}}$ in the galaxy mass range, which agrees well with observations. On the other hand, the value of the constant $\rho_{\mathrm{c}} r_{\mathrm{b}}$ predicted by Eq. (25) is lower approximately by a factor of seven than the observed value. The contradiction may be obviated if we assume that supposition (23) of the value of $\langle\alpha\rangle$ is not true. Indeed, Eq. (23) implies that $\langle\alpha\rangle$ has the highest possible value. This is not necessarily so; moreover, there are some reasons to believe that the mean-square-root angular momentum of the particles of DM halos is fairly small (Baushev 2011). According to Eq. (22), $\rho_{\mathrm{c}} r_{\mathrm{b}}$ is inversely proportional to $\langle\alpha\rangle$. If we insert

$\langle\alpha\rangle=\frac{1}{28} \sqrt{G M_{\mathrm{vir}} R_{\mathrm{vir}}}$

instead of Eq. (23) into (22), we obtain the dependence of $\rho_{\mathrm{c}} r_{\mathrm{b}}$ on $M_{\text {vir }}$ in excellent agreement with observations (the dashed line in Fig. 3).

It is important to underline that concluding about the constancy of the multiplication $\rho_{\mathrm{c}} r_{\mathrm{b}}$ for the galaxy mass objects is an inherent property of the moderate-relaxation model and does not depend on the choice of constants in Eqs. (23) and (32). For more massive halos $\left(M_{\mathrm{vir}} \geq 10^{13} M_{\odot}\right)$ the model predicts an even weaker dependence of $\rho_{\mathrm{c}} r_{\mathrm{b}}$ on $M_{\mathrm{vir}}$; however, the model itself is hardly adequate for objects this massive. Very small ha$\operatorname{los}\left(M_{\mathrm{vir}}<10^{6} M_{\odot}\right)$ should have $\rho_{\mathrm{c}} r_{\mathrm{b}} \propto M_{\mathrm{vir}}^{1 / 3}$, that is, $\rho_{\mathrm{c}} r_{\mathrm{b}}$ is not quite constant anymore. However, the dependence remains rather weak.

\section{Conclusion}

Thus assuming moderate relaxation in the formation of dark matter halos invariably leads to density profiles that match those observed in the central regions of galaxies. The profile is insensitive to the initial conditions. It has a central core; in the core area the density distribution behaves as an Einasto profile with a low index $(n \sim 0.5)$. At larger distances it has an extended region with $\rho \propto r^{-2}$. The multiplication of the central density by the core radius is almost independent of the halo mass.

This is exactly the shape that observations suggest for the central region of galaxies. On the other hand, it does not fit the galaxy cluster profiles. This implies that the relaxation of huge objects $\left(M_{\text {vir }}>10^{13} M_{\odot}\right.$, galaxy clusters $)$ is violent. However, it is moderate for galaxies or smaller objects.

The most plausible explanation of this fact is that the concentrations $c_{\text {vir }}$ of small halos are much higher. As we showed, for the relaxation to be violent, the energies of a significant part of the particles need to change by a factor $\sim c_{\text {vir }}$ with respect to the initial values (so that these particles have $r_{0} \simeq 0$ and form the cusp). Consequently, for galaxy clusters the relaxation is violent if the energies of the particles decrease by a factor 3-5 ( $c_{\text {vir }}=3-5$ for these objects $)$. Such a change seems possible even in a collisionless system. However, $c_{\text {vir }} \sim 15$ even for massive galaxies and can be much higher for dwarf objects. Then the violent relaxation claims that the energies of a significant fraction of the particles change by a factor 15-20: this requirement is quite strong. Probably, some other factors, such as the baryon component, also influence the intensity of the relaxation. This question merits a more detailed consideration.

Acknowledgements. Financial support by Bundesministerium für Bildung und Forschung through DESY-PT, grant 05A11IPA, is gratefully acknowledged. BMBF assumes no responsibility for the contents of this publication. We acknowledge support by the Helmholtz Alliance for Astroparticle Physics HAP funded by the Initiative and Networking Fund of the Helmholtz Association.

\section{Appendix A: A convolution of two functions: how does one transform Eq. (18) into (20)?}

We consider a convolution of two functions $\int g_{1}(x) g_{2}(x, y) \mathrm{d} x$ where $g_{1}(x)$ is finite, that is, $g_{1}(x)$ differs noticeably from zero only in a narrow interval $x \in\left[x_{1}, x_{2}\right]$, and $g_{2}(x, y)$ depend only slightly on $x$ at this interval for any given $y$. Then we may roughly estimate

$\int g_{1}(x) g_{2}(x, y) \mathrm{d} x \simeq g_{2}(\langle x\rangle, y) \int g_{1}(x) \mathrm{d} x$,

where $\langle x\rangle$ is the value of $x$ averaged over $\left[x_{1}, x_{2}\right]$. Indeed, Eq. (A.1) becomes exact if the width of $g_{1}(x)$ is negligible $\int \delta\left(x-x_{0}\right) g_{2}(x, y) \mathrm{d} x=g_{2}\left(x_{0}, y\right)$. It is also exact if $g_{2}(x, y)$ does not change at all, when $x$ runs between $x_{1}$ and $x_{2}$ : $\int g_{1}(x) g_{2}(y) \mathrm{d} x=g_{2}(y) \int g_{1}(x) \mathrm{d} x$. If $g_{2}(x, y)$ depends on $x$ at $\left[x_{1}, x_{2}\right]$, Eq. (A.1) is an approximation. However, our prime interest here is the accuracy of transformation (18) into (20) with the help of Eq. (A.1). We show below that the approximation is quite good in this case.

First of all, we should determine the best way to find $r_{\mathrm{c}}$ (see Eq. (19)), that is, to average $\frac{\sqrt{2|\phi(0)|}}{\alpha\left(r_{0}\right)}$ over the halo. Since we are interested in the very central region of the halo, we consider the case when $r \rightarrow 0$. The Dawson function $D(x) \simeq x$ if $x$ is small, and we obtain from Eq. (18)

$\rho_{\mathrm{c}}=\frac{1}{2 \pi} \int_{0}^{\infty} \frac{f\left(r_{0}\right)}{\alpha\left(r_{0}\right) T\left(r_{0}\right)} \frac{\sqrt{2|\phi(0)|}}{\alpha\left(r_{0}\right)} \mathrm{d} r_{0}$.

Dividing this equation by $(21)$, we obtain

$\frac{1}{r_{\mathrm{c}}}=\frac{\int_{0}^{\infty} \frac{f\left(r_{0}\right)}{\alpha\left(r_{0}\right) T\left(r_{0}\right)} \frac{\sqrt{2|\phi(0)|}}{\alpha\left(r_{0}\right)} \mathrm{d} r_{0}}{\int_{0}^{\infty} \frac{f\left(r_{0}\right) \mathrm{d} r_{0}}{\alpha\left(r_{0}\right) T\left(r_{0}\right)}}$. 


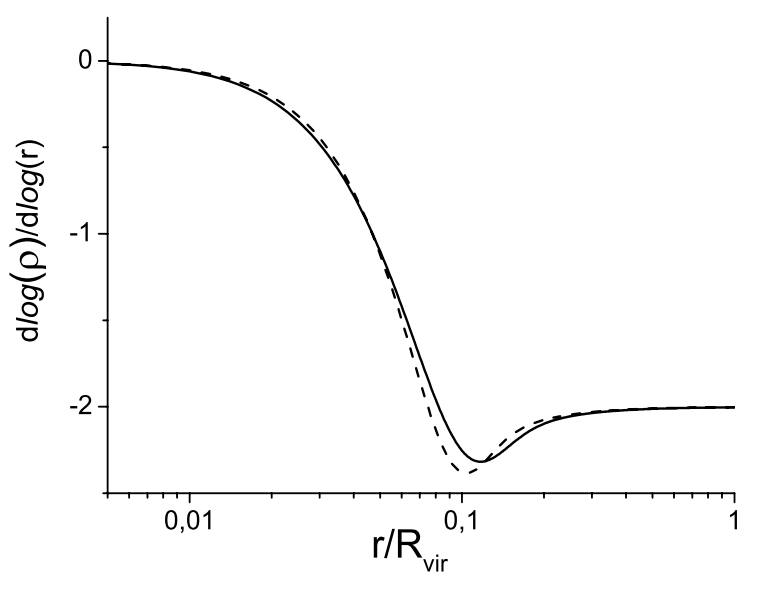

Fig. A.1. Shapes of density profiles calculated with the help of the exact Eq. (18) (solid line) and approximation (20) (dashed line, see the Appendix for details).

This equation can be considered as a sort of averaging of function $\frac{\sqrt{2|\phi(0)|}}{\alpha\left(r_{0}\right)}$ over the halo with the use of $\frac{f\left(r_{0}\right)}{\alpha\left(r_{0}\right) T\left(r_{0}\right)}$ as the weighting function. This method of averaging yields the best fitting of Eq. (18) in the halo center.

To estimate the accuracy of transformation (18) into (20), we may consider a more or less realistic model of functions $f\left(r_{0}\right)$, $\alpha\left(r_{0}\right), T\left(r_{0}\right)$, and then to compare the density profiles obtained with the help of Eqs. (18) and (20). We assume that $f\left(r_{0}\right)$ has a Gaussian shape

$$
\begin{cases}f\left(r_{0}\right) \propto \exp \left(-\frac{\left(r_{0}-a\right)^{2}}{2 \sigma^{2}}\right), & r_{0}>0.1 R_{\mathrm{vir}} \\ f\left(r_{0}\right)=0, & r_{0}<0.1 R_{\mathrm{vir}},\end{cases}
$$

where $a=0.7 R_{\mathrm{vir}}, \sigma=0.2 R_{\mathrm{vir}}$. The parameters are chosen so that the total final energy of the halo is approximately equal to the initial one, $f\left(r_{0}\right)$ is finite, as described at the end of the Energy distribution section, and the region $\frac{2 R_{\mathrm{vir}}}{c_{\mathrm{vir}}}$ falls approximately outside the $3 \sigma$ area, if $c_{\text {vir }} \sim 15$, which is a typical value for galaxies. $T\left(r_{0}\right)$ is mainly defined by the particle motion near the apocenter (Baushev 2013a). Since $M\left(r_{0}\right) \simeq M_{\mathrm{vir}}$ if $r_{0} \sim R_{\text {vir }}$, we may assume that $T\left(r_{0}\right) \propto \sqrt{r_{0}}$, as in the case of a point mass $M_{\text {vir }}$. The same reasoning allows us to assume by analogy with Eqs. (23) and (32) that $\alpha\left(r_{0}\right) \propto \sqrt{M\left(r_{0}\right) r_{0}} \propto \sqrt{r_{0}}$, and that $\sqrt{2\left(\phi\left(r_{0}\right)-\phi(0)\right)} \simeq \sqrt{2|\phi(0)|}$. A normalization of $f\left(r_{0}\right)$, $\alpha\left(r_{0}\right)$, and $T\left(r_{0}\right)$ is not significant, since we are interested in the profile shape, and we plot it in $\log \rho / \log r$ coordinates. However, we choose a proper value of $\sqrt{2|\phi(0)|}$ to obtain a desirable value of $r_{\mathrm{c}}$ according to Eq. (A.3). We chose $r_{\mathrm{c}}=0.05 R_{\mathrm{vir}}$, exactly as in Fig. 2.

Figure A.1 represents the density profiles calculated for this model with the help of exact Eq. (18) (solid line) and approximation (20) (dashed line). Clearly, the deviations are quite small, especially in the halo center and at large radii. Moreover, approximation (20) has the same shape as the exact solution, that is, the same Einasto-like profile in the center, the same core radius, and the same $\rho \propto r^{-2}$ region. Consequently, the conclusions of this paper are valid for the exact solution (18) as well. Thus the approximation of Eq. (18) by (20) is quite accurate for realistic models of halo parameters.

\section{References}

Baushev, A. N. 2011, MNRAS, 417, L83

Baushev, A. N. 2012, MNRAS, 420, 590

Baushev, A. N. 2013a, ApJ, 771, 117

Baushev, A. N. 2013b, Astropart. Phys., accepted [arXiv: 1312.0314]

Baushev, A. N. 2014, ApJ, 786, 65

Binney, J., \& Tremaine, S. 2008, Galactic Dynamics: 2d edn. (Princeton University Press)

Burkert, A. 1995, ApJ, 447, L25

Carney, B. W., \& Latham, D. W. 1987, in Dark matter in the Universe, eds. J. Kormendy, \& G. R. Knapp, IAU Symp., 117, 39

Chemin, L., de Blok, W. J. G., \& Mamon, G. A. 2011, AJ, 142, 109

Cooray, A., \& Sheth, R. 2002, Phys. Rep., 372, 1

de Blok, W. J. G., \& Bosma, A. 2002, A\&A, 385, 816

de Blok, W. J. G., McGaugh, S. S., \& Rubin, V. C. 2001, AJ, 122, 2396

Diemand, J., \& Kuhlen, M. 2008, ApJ, 680, L25

Diemand, J., Madau, P., \& Moore, B. 2005, MNRAS, 364, 367

Diemand, J., Kuhlen, M., \& Madau, P. 2007, ApJ, 667, 859

Donato, F., Gentile, G., Salucci, P., et al. 2009, MNRAS, 397, 1169

Evans, N. W., \& Collett, J. L. 1997, ApJ, 480, L103

Gentile, G., Salucci, P., Klein, U., \& Granato, G. L. 2007, MNRAS, 375, 199

Gorbunov, D. S. \& Rubakov, V. A. 2010, Introduction to the Early Universe theory. Vol. 2: Cosmological perturbations. (Moscow: LKI publishing house) Governato, F., Zolotov, A., Pontzen, A., et al. 2012, MNRAS, 422, 1231

Hansen, S. H., Moore, B., Zemp, M., \& Stadel, J. 2006, J. Cosmol. Astropart, 1, 14

Hayashi, E., Navarro, J. F., Taylor, J. E., Stadel, J., \& Quinn, T. 2003, ApJ, 584, 541

Klypin, A., Zhao, H., \& Somerville, R. S. 2002, ApJ, 573, 597

Klypin, A., Prada, F., Yepes, G., Hess, S., \& Gottlober, S. 2013, MNRAS, submitted [arXiv: 1310.3740 ]

Kormendy, J., \& Freeman, K. C. 2004, in Dark Matter in Galaxies, eds. S. Ryder, D. Pisano, M. Walker, \& K. Freeman, IAU Symp., 220, 377

Kuhlen, M., Weiner, N., Diemand, J., et al. 2010, J. Cosmology Astropart. Phys., 2,30

Landau, L. D., \& Lifshitz, E. M. 1980, Statistical physics, Pt.1, Pt.2 (Oxford: Pergamon Press)

Lynden-Bell, D. 1967, MNRAS, 136, 101

Marchesini, D., D’Onghia, E., Chincarini, G., et al. 2002, ApJ, 575, 801

Marochnik, L. S., \& Suchkov, A. A. 1984, The Galaxy (Moscow: izdatel'stvo Nauka) [in Russian]

Moore, B., Quinn, T., Governato, F., Stadel, J., \& Lake, G. 1999, MNRAS, 310, 1147

Navarro, J. F., Ludlow, A., Springel, V., et al. 2010, MNRAS, 402, 21

Neto, A. F., Gao, L., Bett, P., et al. 2007, MNRAS, 381, 1450

Oh, S.-H., de Blok, W. J. G., Brinks, E., Walter, F., \& Kennicutt, Jr., R. C. 2011, AJ, 141, 193

Okabe, N., Zhang, Y.-Y., Finoguenov, A., et al. 2010, ApJ, 721, 875

Planck Collaboration XVI. 2014, A\&A, in press DOI: $10.1051 / 0004-6361 / 201321591$

Power, C., Navarro, J. F., Jenkins, A., et al. 2003, MNRAS, 338, 14

Quinlan, G. D. 1996, New Astron., 1, 255

Rubin, V. C., Thonnard, N., \& Ford, Jr., W. K. 1978, ApJ, 225, L107

Salucci, P., Lapi, A., Tonini, C., et al. 2007, MNRAS, 378, 41

Sofue, Y., \& Rubin, V. 2001, ARA\&A, 39, 137

Stadel, J., Potter, D., Moore, B., et al. 2009, MNRAS, 398, L21

Tollerud, E. J., Beaton, R. L., Geha, M. C., et al. 2012, ApJ, 752, 45

Wang, J., Frenk, C. S., Navarro, J. F., Gao, L., \& Sawala, T. 2012, MNRAS, 424, 2715 